THE capacity of those individuals who survive infectious diseases to become immune to further attack was recognised early in history, and for several hundred years attempts have been made to exploit this phenomenon in medicine. It is nearly one hundred years since Behring showed that the substances which are responsible are present in the blood of the immune animal and named them antibodies, but it is less than twenty years since advances in knowledge of protein chemistry made it possible to isolate and study the structure of these remarkable molecules. Their fascination lies in the ability of almost identical molecules to combine specifically with an apparently endless range of different substances and the recognition that this phenomenon is essential for the survival of all species of vertebrates. The growing realisation that a related phenomenon may be the basis of cellular recognition and therefore be an important feature of differentiation has put the antibody molecule in a key position in biological research. Here for the first time, the variable region of antibody molecules offers a chemical basis for one aspect of the infinite variety of living things. The genetic origin of this very wide range of protein structures found in all individuals remains a mystery, but one likely to be solved soon.

Even so, it is surprising to find in this book just how much information has been acquired in so short a time. The emphasis throughout the book is on the structure of the immunoglobulins, the proteins which carrv the antibody activity. The chemical structures which

\section{Antibody molecule}

\section{R. R. Porter}

The Antibody Molecule. (Immunology: An International Series of Monographs and Treatises.) By Alfred Nisonoff, John E. Hopper and Susan B. Spring. Pp. xiv +542 . (Academic: New York, San Francisco and London, August 1975.) $\$ 34.50 ; £ 16.55$.

define the division of the immunoglobulins into class and subclass, type and subtype, the different polymorphic forms, and the amino acid sequence changes found in the variable regions are all well described. Most of this work is soundly based, and although much remains to be investigated sufficient detailed study has been carried out on the immunoglobulins of several species to provide a body of knowledge which is now the basis for present investigations on the genetics, the control of synthesis and cellular origins of immunoglobulin and on many other aspects of immunity.

The authors have written an advanced monograph in which a mass of complex data has been presented in a well ordered and comprehensible manner. Sufficient detail is given to make this book an excellent source of reference for those working in the field, but it never obscures the main themes. Ii should be of equal value for the many biologists with a peripheral interest in the subject. The book has been published quickly: most of the X-ray crystallographic studies of the past year or two are described and a most informative coloured plate has been included of the model of the $F a b$ fragment of a human myeloma protein. Enough experimental detail will be found to support the results described and to explain, for example, the discovery of the still somewhat obscure idiotypic specificities of antibodies.

Most of the work described is of immediate interest, but some, such as the early attempts to define the relative contribution of the heavy and light chain of antibodies to the structure of the combining site, have a dated flavour and already seem to have been superseded by subsequent knowledge of the chemistry and crystallographic studies of the variable regions. Succinct accounts are included of the brief histories of most of the topics discussed and are incomplete only in the overmodest absence of references to the senior author. Careful reading of the extensive bibliographies at the end of each chapter is necessary to appreciate how large a contribution to our knowledge of antibody structure has been made by Dr Nisonoff.

This book gives a clear account of our factual knowledge of the structure of antibodies and of its biological significance. It is likely to remain of enduring value for some years to come.
WE have been here many times before-from early man and increasing plunder of wildlife to population explosion and pollution-but this is a very readable addition to the considerable clutch of similar books.

The detailed effects of man's impact on wildlife (and vice versa) are discussed in convenient form. There are chapters on plants and topography, the sea, marine mammals, birds, insects, and other invertebrates, followed by one on "Conservation Profit and Loss" and a rather curious bibliography for further reading.

The authoritative and lively style is to be welcomed. Not so, instances

\section{Man and wildlife}

Man and Wildlife. (Biology and Environment.) By L. Harrison Matthews. Pp. $129+16$ plates. (Croom Helm: London, September 1975.) $£ 4.95$.

of what seems to be prejudice leading to dubious generalisation and emotive references out of tune with the character of the book. As an example, "superficial" wildlife films on television as well as bird watchers with their "little" lists are castigated with- out acknowledgement that herein may lie the genesis of funds for holding open the conservation options for future generations - to purchase Wash and Lake Nakuru mud, and to pay for 'Operation Tiger' and education all over the world.

The Publishers' note on the wrapper expressing some hope for wildlife's future is clearly not endorsed by the author in his concluding chapter which omits adequate analysis of the conservation effort. Yet if we are to accept that all hope is lost at this stage. much of the raison d'être of civilised man is lost also. 